\title{
A novel keratinocyte delivery system to burn wounds
}

\section{B. ter Horst ${ }^{\mathrm{a}, \mathrm{b}}$, G. Chouhan ${ }^{\mathrm{a}}$, N.M. Moiemen ${ }^{\mathrm{b}}$, L.M. Grovera}

a. University Hospitals Birmingham NHS Foundation Trust at Queen Elizabeth Hospital Birmingham, Burns Centre. Mindelsohn Way, B15 2TH, Birmingham, United

Kingdom. B. School of Chemical Engineering, University of Birmingham, Edgbaston, B15 2TT, United Kingdom.

† Corresponding author.: Britt ter Horst, email: bxt564@bham.ac.uk

\section{Introduction}

To achieve faster re-epithelialisation of burn wounds, the original autologous keratinocyte culture and transplantation technique was introduced over 3 decades ago. Application types of keratinocytes transplantation have improved from cell sheets to single-cell solutions delivered with a spray system. Nevertheless, some shortcomings of the suspension application technique have yet to be addressed. For example, an uneven wound bed (combined with a curved body contour), can result in uneven spreading of the cell suspension or dripping off the wound bed (Fig 1c). Tailored spray devices can play an important role in improving spray cell delivery to burn wounds.

\section{Objectives}

The aim of this study is to develop an effective carrier system for spray cell transplantation to burn wounds and provide a novel spray assessment method.

\section{Methods}

Spray characteristics of a potential cell carrier (Gellan ${ }^{\circledR}$ hydrogel) was compared to a control liquid. Spray application through an airbrush (Fig. 1b) with standardized nozzle diameter, nozzle to surface angle, distance and delivered air pressure was performed.

Percentage of surface area covered (\%coverage), droplet size, droplet size distribution (relative span factor) and spread rate were determined utilizing water-sensitive paper (WSP), measuring $26 \times 76 \mathrm{~mm}$, on a flat and tilted receiving surface (Fig2a). Sprayed WSP was scanned and analysed with Image J software. Cell viability was assessed with live/dead staining following gellan hydrogel encapsulation and spraying.

\section{Results}

Percentage coverage: Following spray application of $25 \mu \mathrm{l}$ and $50 \mu \mathrm{l}$ fluid volume, water showed higher \%coverage (41.0\%, range $25.5-69.1 \%)$ compared to Gellan $(24.8 \%$, range $7.7-49,1 \%)$ for all sprayed volumes, although the differences are not significant (Fig2b).

\section{Fig 2a: water-s

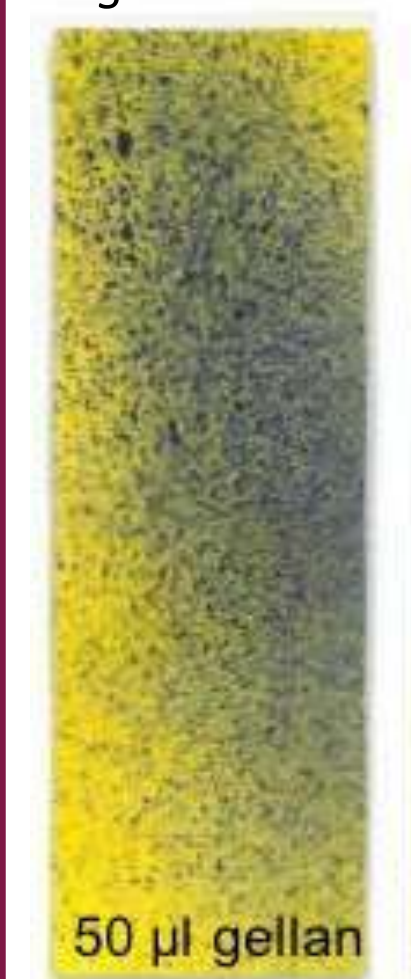

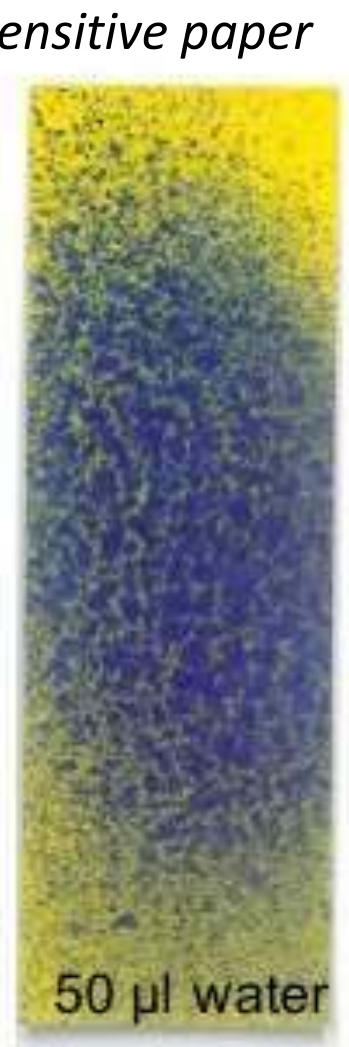

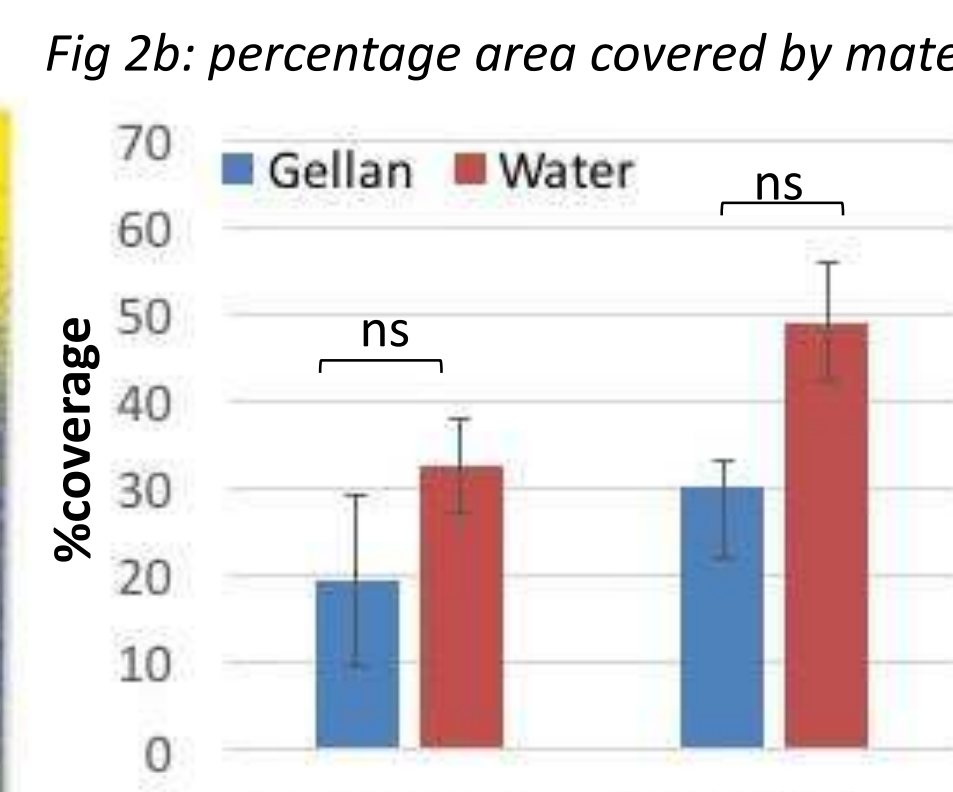

volume $25 \mu \mathrm{l} \quad$ volume $50 \mu \mathrm{l}$ $\mathrm{p}=0.275(95 \% \mathrm{Cl}[-40.84,14.23](25 \mu \mathrm{l})$ $\mathrm{p}=0.654(95 \% \mathrm{Cl}[-33.24889,-4.89256](50 \mu \mathrm{l})$ Error bars represent SEM

Non-significant correlation between sprayed volume and \%coverage for gellan $(p=0.301,95 \% \mathrm{Cl}$ : 33.09, 11.86; Pearson correlation 0.820$)$ and water $(p=0.065$, Cl95\%: -34.066, 1.308; Pearson correlation 0.723). (Fig2b)

\section{Spread rate:}

Fig 1a: comparison of dyed gellan fluid gel (left), quiescent gellan gel (middle) and sodium chloride (right) droplets to porcine skin indicates a low rate of spreading of gellan. Less run-off was seen with gellan hydrogel spray (Fig1c) compared to control (Fig1d).

Fig 1a-d:

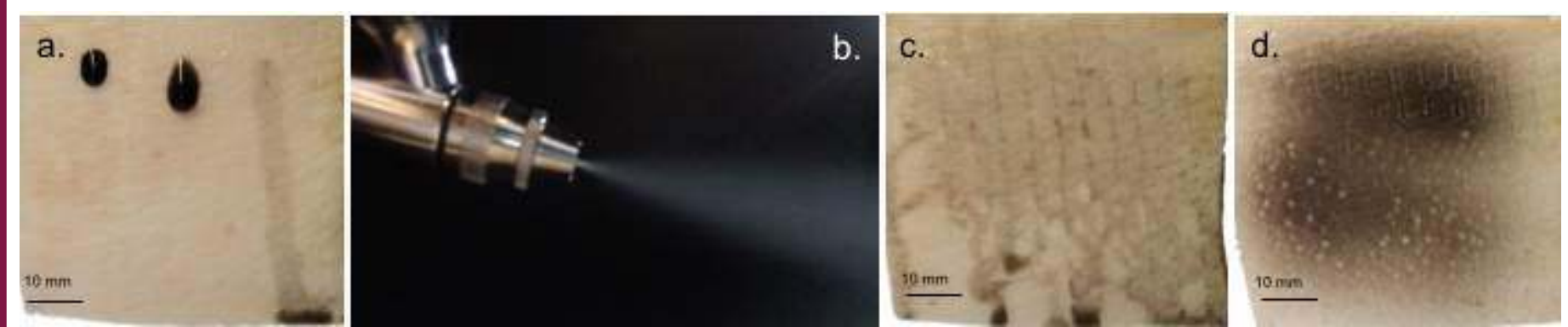

Hydrogel droplets of known volumes $(3,4,5,10 \mu \mathrm{l})$ applied to flat and tilted WSP were allowed to dry. Gellan hydrogel preserved structural stability better than water as the expanded area of stained WSP is 3.1 times (flat) and 2.2 times (tilted) less than water.

\section{Spray distribution:}

A more uniform droplet size distribution and potentially less volume dependent distribution of sprayed gellan is reflected by the lower mean relative span factor of gellan (mean 8.57, SD 4.15) compared to water (mean 11.84, SD 4.87), however, these differences are not significant (Fig3a/b)

\section{Figure $3 a / b$ : mean relative span factor}

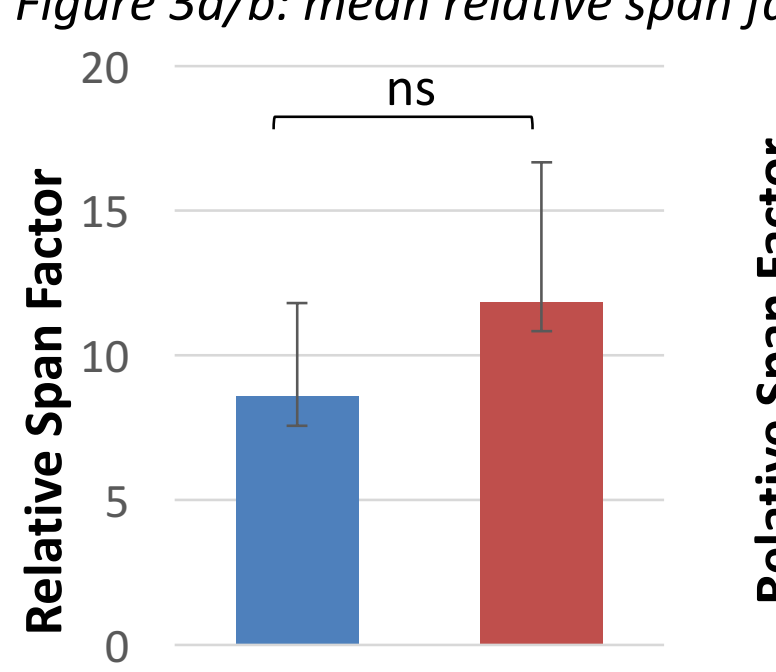

gellan water

$p=0.457[95 \% \mathrm{Cl}:-8.77,2.23]$

\section{Cell viability:}

Fluorescence cell viability assay of encapsulated human dermal fibroblasts (HDF) is shown for day 1 and day 3 following spraying (live cells green, dead cell red)[Fig $4 a / b]$. High cell viability for up to 14 days post encapsulation and spraying (Fig 4d). Cells encapsulated in gellan hydrogel remain viable, attach to the bottom of the well and seem to migrate out of the gel (Fig 4c).

Figure 4a-d: cell viability
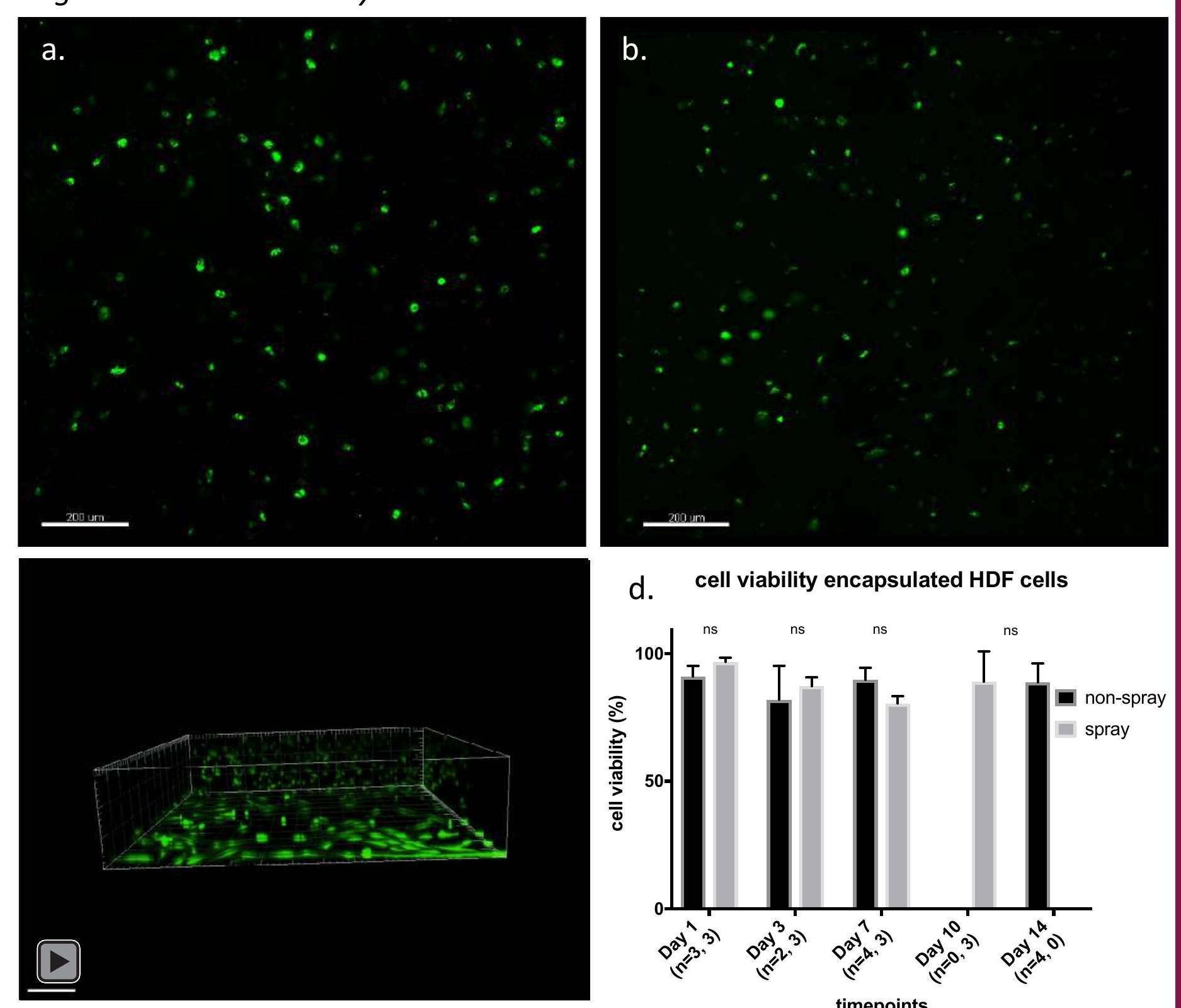

d. cell viability encapsulated HDF cells

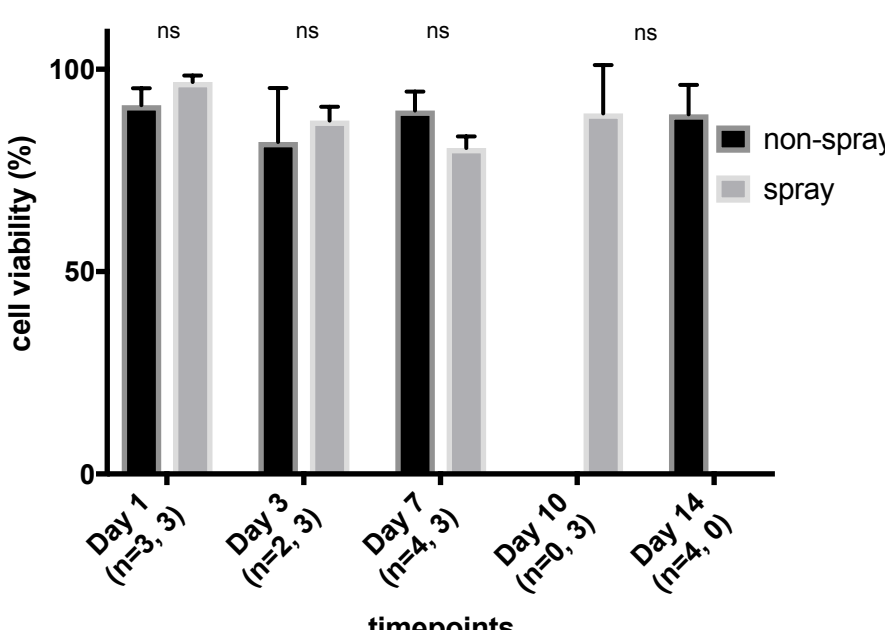

\section{Conclusion}

Future approaches of cell transplantation involve spray devices, but optimisation of application technique and carrier type is necessary. Watersensitive paper is an useful addition to assess spray characteristics. Preliminary in vitro results from our studies show that cells tolerate gellan hydrogel encapsulation and spray delivery. Spray assessment of gellan demonstrates limited runoff and spreading to the receiving surface and a more uniform droplet distribution compared to water. Therefore, gellan hydrogel seems a promising candidate for both cell encapsulation and spray application. 